\title{
REVISÃO INTEGRATIVA: FISIOTERAPIA EM TERAPIA INTENSIVA NEONATAL
}

Rúbia Mara Giacchini Kessler $^{1}$

Doutoranda em Oncologia pelo A.C. Camargo (2018) Mestre em Saúde e Gestão do Trabalho pela Universidade do Vale do Itajaí (2007), fez graduação em Fisioterapia pela Fundação Universidade Regional de Blumenau (1998) e especialização em Fisioterapia Cardiorrespiratória pela Universidade Tuiuti do Paraná (2002). Atualmente é professor titular da Universidade do Vale do Itajaí. E-mail: rubia@univali.br

\section{Thalis Vagetti Lee} Barduzzi Netto ${ }^{2}$

Residência Multiprofissionbal em Atençao Básica -

UNIVALI(2018-2019) Graduação em Fisioterapia pela

Universidade do Vale do

Itajaí(2017). E-mail:

thalislee@gmail.com

\section{Lauanna Pael Alcará ${ }^{3}$}

Graduada em Fisioterapia pela Universidade do Vale do Itajaí (2018). Bolsista/Participante do Projeto de Extensão:

Atividade/Terapia Assistida por Animais (A/TAA) como

Alternativa de Humanização dos Cuidados em Saúde da Universidade do Vale do Itajaí (Univali). Possui graduação em Comunicação Social -

Habilitação em Jornalismo pela Universidade Federal de Mato Grosso do Sul (2010)

E-mail: thalislee@gmail.com

\author{
INTEGRATIONAL REVIEW: PHYSIOTHERAPY IN NEONATAL \\ INTENSIVE CARE UNIT
}

\section{RESUMO}

O objetivo: Identificar a indicação científica sobre início da intervenção fisioterapêutica em UTIs neonatais. Metodologia: Revisão integrativa com estudos em Português, Inglês e Espanhol publicados nos últimos 10 anos (i.e.: desde 2006) nas bases eletrônicas Scielo e CAPES. Foram selecionados 81 artigos, destes, 50 foram excluídos. Resultados: Dos artigos analisados, 9 apresentaram descrição para início da intervenção fisioterapêutica. Foram apresentados 4 principais fundamentos de indicação: prescrição médica; somente após 72 horas de vida; nas primeiras 72 horas de vida; após 12 horas de aplicação do surfactante exógeno. Conclusão: O início da fisioterapia para recém-nascidos internados em UTIs precisa de mais estudos experimentais para traçar critérios claros de que momento o fisioterapeuta deve iniciar a intervenção.

PALAVRAS-CHAVE: UTI neonatal; Neonatos; Lactentes prematuros.

\section{ABSTRACT}

Objective: Identify the scientific indication about the beginning of the physiotherapeutic intervention in neonatal ICUs. Methodology: Integrative review with studies in Portuguese, English and Spanish published in the last 10 years (since 2006) in the databases Scielo and CAPES. Initially, 81 articles were selected, of which 50 were excluded. Results: Of the articles analyzed, 9 presented a description for the beginning of the physiotherapeutic intervention. Four main reasons for indication were presented: medical prescription; only after 72 hours of life; during the first 72 hours of life; after 12 hours of application of the exogenous surfactant. Conclusion: The onset of physiotherapy for newborns hospitalized in ICUs requires more experimental studies to draw clear criteria of when the physiotherapist should initiate the intervention.

KEYWORDS: Neonatal ICU; Neonates; Premature infants.

\section{INTRODUÇÃO}

Os recém-natos prematuros, conforme Hackbarth et al. (2015), são definidos pela Organização Mundial da Saúde (OMS) todos aqueles oriundos de gestações por períodos inferiores há 37 semanas, podendo ser classificados em

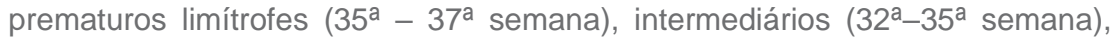




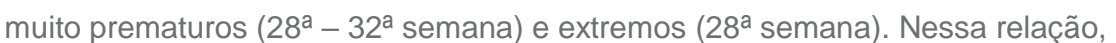
segundo Balbi, Carvalhaes e Parada (2016), o Brasil está entre as 10 nações com maior número de nascimentos pré-termos e em conformidade com Guinsburg e Almeida (2016), seria o 16ํem número de óbitos decorrentes de complicações da prematuridade. Esses lactentes são mais propensos a consequências como enterocolite necrotizante, hemorragia intraventricular, problemas respiratórios, hipertensão pulmonar persistente e ainda frequente déficit de desenvolvimento (BALBI, CARVALHAES e PARADA, 2016).

Santos, Meira e Pereira (2016) afirmam que alterações do sistema cardiovascular também são frequentes em neonatos prematuros, principalmente aqueles de muito baixo peso, sendo que estas ocorrem desde as primeiras horas de vida por causas diversas. Dessa forma, para o aumento da sobrevida entre os recém-nascidos de baixo peso e/ou prematuros (RNPT), torna-se imprescindível que estes tenham acompanhamento do desenvolvimento e avaliações específicas mais precocemente e que, se necessário, intervenções sejam realizadas para que tenham qualidade de vida semelhante aos nascidos a termo e com peso adequado.

A Fisioterapia Neonatal atua diretamente nesses fatores com aplicação de recursos relacionados à manutenção da permeabilidade de vias aéreas, processo de instituição e gerenciamento da Ventilação Mecânica (VM); suporte ventilatório não invasivo; oxigenioterapia e mobilização precoce com utilização de recursos cinético funcionais, podendo ser classificadas como condutas de fisioterapia respiratória e/ou motora.

Apesar da atuação da fisioterapia na UTI neonatal estar consolidada, ainda não há consenso na literatura do momento em que o fisioterapeuta pode iniciar a intervenção motora e/ou respiratória.

Assim, o objetivo deste artigo consiste na realização de uma revisão integrativa dos estudos publicados sobre a intervenção fisioterapêutica nas Unidades de Terapia Intensiva Neonatais, desde 2006 até o ano de 2016, para identificar qual a indicação científica sobre o início da intervenção fisioterapêutica em UTIs neonatais.

Neste sentido, foram estabelecidos 4 objetivos específicos: 1. Caracterizar os estudos encontrados sobre o tema (número de artigos, ano de publicação, revistas de publicação, local de publicação e tipo de estudo); 2. Identificar quais os descritores mais utilizados nos estudos; 3. Apresentar quando os autores sugerem o início da intervenção fisioterapêutica; 4. Comparar os estudos disponíveis na literatura, sobre qual o melhor momento para iniciar as intervenções fisioterapêuticas em recém-nascidos nas UTIs.

\section{METODOLOGIA}

Para começar esta análise, foi definida uma série de critérios para à seleção prévia da literatura: (1) a pesquisa foi realizada com estudos originais escritos em Português, Inglês e Espanhol devido à maior acessibilidade linguística; (2) foram selecionados estudos de caráter experimental, revisões sistemáticas e estudos de caso realizados nos últimos 10 anos de investigação (i.e.: desde 2006); (3) e em relação à sua natureza (como fontes de informação apropriadas) foram eleitos artigos científicos de revistas, conferências e documentos pertencentes às coleções online publicadas nas bases de dados eletrônicas científicas. A consulta dos estudos procedeu-se nas bases eletrônicas: Scielo e CAPES. Concedeu-se prioridade aos trabalhos publicados em revistas 
indexadas, reconhecidas por suas devidas contribuições científicas e/ou os de acesso gratuito, garantindo disponibilidade a uma maior quantidade de investigações.

Em seguida, foram definidas as palavras chaves que guiaram a pesquisa: Português ("Neonatos", "Lactente Prematuro", "Prematuridade Neonatal", "Recém-Nascido Prematuro", "Unidade de terapia intensiva em neonatal", "Centros de terapia intensiva para recém-nascidos", "Unidades de terapia intensiva para recém-nascidos", "Serviço hospitalar de fisioterapia"); inglês ("Newborn", "Intensive care units neonatal", "Physical therapy department hospital"); e espanhol ("Unidades de cuidado intensivo neonatal", "Recién nacido", "Neonatos"). Seguidamente, os critérios de exclusão estabelecidos foram: (a) todos os documentos originais publicados em línguas para as quais não se possui acessibilidade; (b) estudos onde não seja abordada a intervenção fisioterapêutica diretamente com neonatos; (c) e conteúdo que não se relacionam com UTIs neonatais especificamente.

Após a localização das publicações que cumpriram os requisitos, os artigos foram organizados em uma planilha do Excel, sendo divididos em duas grandes dimensões: primeiramente, é apresentada a constituição dos dados relativos a cada publicação (identificação dos autores e categoria profissional, ano de publicação, fonte editorial, título, fator de impacto, origem e instituição que suportou o estudo, e o tipo e natureza do trabalho (experimental vs. teórico); a segunda dimensão abrange as informações características das pesquisas: objetivo do estudo, caracterização da amostra (grupo etário, gênero, diagnóstico), palavras-chave, categorização do tema e das perturbações em estudo, assim como o desenvolvimento das intervenções e sua aplicação (fisioterapia respiratória e/ou motora, periodicidade e frequência das sessões, em grupos vs. individual, com ou sem grupo controle, os efeitos e a sua manutenção, os instrumentos de avaliação utilizados). 


\section{Quadro 1 - Fluxograma do processo de obtenção da literatura.}

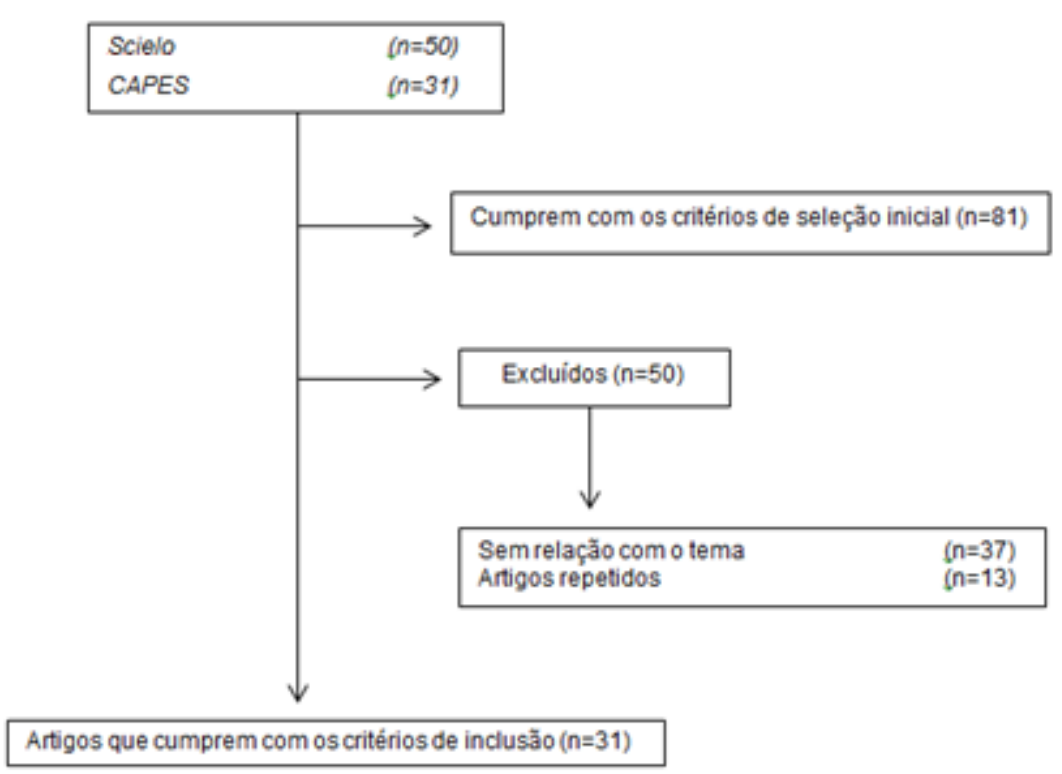

Fonte: Autores, 2017.

Primordialmente, o quadro 1 demonstra o filtro das buscas em sua totalidade $(n=81)$, as respectivas bases de dados utilizadas (Scielo $n=50$, CAPES $n=31$ ), os artigos excluídos $(n=50)$ com seus devidos motivos (sem relação com o tema $n=37$ e repetição nos periódicos $n=13$ ) e por fim, os artigos que cumprem com os critérios de inclusão ( $n=31)$.

\section{RESULTADOS}

Conceitualmente o termo qualidade de vida, em virtude de sua complexidade Podemos observar no quadro 1 que o periódico que publicou o maior número de artigos com a temática da atuação fisioterapêutica em Unidade de Terapia Intensiva em Neonatologia, foi a Scientific Eletronic Library Online (Scielo), totalizando 50 artigos.

Dos 81 artigos que cumprem os critérios de seleção inicial, 50 foram excluídos. Os motivos para exclusão foram: não ter relação com o tema $(n=37)$; e serem repetidos $(n=13)$. Apesar de apresentarem os descritores, durante a análise destes artigos evidenciamos algumas faltas correlacionais com a temática a ser explorada. Havia pesquisas em que a população amostral não contextualizava com o objetivo de nossa busca, outras abordavam as intervenções fisioterapêuticas fora das Unidades de Terapia Intensiva, bem como condutas que não são específicas da fisioterapia. Neste último, temos como exemplo o artigo de Almeida e Forti (2007) que aborda a conduta do método Canguru, sendo que este pode ser realizado por outros profissionais. Referente aos demais artigos excluídos ( $n=13)$, observamos que estes estavam se repetindo em ambas as bases de dados (Scielo e CAPES), dessa forma escolhemos a imparidade na busca. 


\section{Artigos Inclusos}

Os 31 artigos que alcançaram aos critérios de inclusão foram tabulados em uma planilha eletrônica do Excel, na qual foram acrescentados dados como o título, ano de publicação, local de publicação, nome dos autores, natureza do trabalho, objetivo do estudo e sua metodologia, bem como a caracterização da amostra (grupo etário, gênero e diagnóstico), palavras-chaves, se especificava critério para iniciar a intervenção fisioterapêutica na UTI neonatal e ainda, quais as condutas realizadas na pesquisa.

Ao se analisar o ano das publicações (2006-2017), constatamos que no período de 2010 o número das produções científicas foi o mais elevado, totalizando 8 artigos, sendo que nos anos de 2006, 2007, 2015 e 2017 constaram apenas um artigo por data.

Em relação aos locais que estes artigos foram publicados, observamos uma totalidade de 12 revistas, sendo uma destas de origem estrangeira e as demais brasileiras (11). Com destaque em publicações, a Revista Brasileira de Fisioterapia/Brazilian Journal of Physical Therapy se sobressaiu, com a exposição de 7 trabalhos. A Revista Paulista de Pediatria e Revista Brasileira de Terapia Intensiva se equivaleram com 5 publicações cada. Dando sequencialmente a continuidade de número de publicações pelas revistas: Fisioterapia em Movimento (4); Revista Brasileira Saúde Materno Infantil e Fisioterapia e Pesquisa (2); e as demais (Jornal de Pediatria, Aguicháu, Revista Brasileira de Crescimento e Desenvolvimento Humano, ConScientiae Saúde, Revista Associação Médica Brasileira e Anna Nery) apresentaram uma publicação cada.

Nesta análise, 9 dos 31 artigos apresentaram descrição de critérios para início da intervenção fisioterapêutica nas UTIs neonatais. Dos 22 artigos que não apresentaram critérios, 10 abordavam fisioterapia respiratória, 5 abordavam sobre fisioterapia motora e os demais sobre questões de avaliação, recomendações e perfis das atuações dos profissionais de fisioterapia. Dessa forma, a configuração com a qual cada pesquisa apresentou seus estudos foi bastante distinta nesses artigos, de maneira que, aqueles que abordaram intervenções apresentaram os objetivos das pesquisas com determinadas condutas frente ao atendimento na UTI neonatal, sem especificar critérios de início, ao invés disso, apenas foi citado que as intervenções seriam realizadas em prematuros hemodinamicamente estáveis, como no estudo de Vignochi, Teixeira e Nader (2010) e também de Martins et al. (2013). Em outros, foram citados a respeito de discussões sobre a atuação fisioterapêutica, entretanto, sem designar qual postura deveria ser adotada. Selestrin et al. (2007) por exemplo, citam que há controvérsias referentes à fisioterapia respiratória no período neonatal, mas em contrapartida afirmam que estudos clínicos demonstraram benefícios da fisioterapia em recém-nascidos pré-termo (RNPT) através da melhora da mecânica pulmonar e redução da variabilidade hemodinâmica desses RNPT, sendo destacado com efeitos terapêuticos benéficos dos procedimentos intervencionistas de fisioterapia neonatal (PIFN), o que corrobora com Antunes et al. (2006) que reforça a importância da assistência fisioterapêutica nos cuidados ao prematuro.

\section{Artigos Selecionados que especificam sobre o início da atuação da fisioterapia}


Verificamos que, destas nove publicações que apresentam critério para início da intervenção fisioterapêutica, 6 se enquadram na classificação de aplicação prática e três no quesito teórico. Apontando os objetivos, tipo de intervenção, variáveis de inclusão, resultados e conclusão dos artigos de aplicação prática, verificamos que Bassani et al. (2016) examinaram a repercussão da técnica de aumento do fluxo expiratório sobre a hemodinâmica cerebral de 40 neonatos prematuros ( $\leq 34$ semanas) com 8-15 dias de vida, clinicamente estáveis, em ar ambiente ou em uso de cateter de oxigênio, utilizando o exame de ultrassonografia com avaliação por dopplerfluxometria cerebral (velocidade do fluxo sanguíneo cerebral e os índices de resistência e pulsatilidade na artéria pericalosa). Estes autores concluíram que a manobra de aumento de fluxo expiratório não afetou o fluxo sanguíneo cerebral em recém-nascidos prematuros clinicamente estáveis, em vista dos resultados apresentados sobre a velocidade de fluxo no pico sistólico $(p=50)$, a velocidade de fluxo diastólico final $(p=0,17)$, a velocidade média de fluxo $(p=0,07)$, o índice de resistência $(p=0,41)$ e o índice de pulsatilidade $(p=0,67)$.

Cabral e Velloso (2014) compararam o efeito de dois protocolos de manuseio mínimo em variáveis fisiológicas (frequência cardíaca, saturação periférica de oxigênio e temperatura axilar de dez em dez minutos) de 40 recém-nascidos, menores de $1500 \mathrm{~g}$, após a terapia com surfactante. Sendo estes, distribuídos em dois grupos e monitorizados seguidamente durante $72 \mathrm{~h}$. Um grupo permaneceu em manuseio mínimo padrão durante as primeiras $12 \mathrm{~h}$ após surfactante, sendo que o outro grupo ficou em manuseio mínimo por $72 \mathrm{~h}$ após surfactante. Esses autores demonstraram que a prática de manuseio mínimo em recém-nascidos de muito baixo peso não apresentou modificação a respeito da estabilidade fisiológica, quando executada durante 12 horas ou 72 horas após administração surfactante, em vista da indiferença demonstrada pelas variáveis estudadas entre os grupos $(p>0,05)$.

Os autores Vignochi, Teixeira e Nader (2010) avaliaram efeitos da fisioterapia aquática na dor por meio do Sistema de Codificação da Atividade Facial Neonatal - NFCS, no ciclo de sono e vigília (escala de avaliação do ciclo de sono e vigília adaptada de Brazelton) e nos parâmetros fisiológicos de 12 recémnascidos com idades gestacionais inferior a 36 semanas. Concluíram que a fisioterapia aquática pode ser um método simples e efetivo na redução da dor e na melhora da qualidade do sono de bebês prematuros em UTI Neonatal em vista da manutenção dos sinais vitais, modificação significativa referente à escala de avaliação do ciclo de sono e vigília e, redução do escore do NFCS de $5,38 \pm 0,91$ para $0,25 \pm 0,46$ após a intervenção.

Antunes et al. (2006) compararam os efeitos da fisioterapia respiratória convencional (FRC) versus aumento do fluxo expiratório (AFE), na saturação periférica de oxigênio $\left(\mathrm{SpO}^{2}\right)$, frequência cardíaca $(\mathrm{FC})$ e na frequência respiratória (FR), de 40 recém-nascidos, sendo o principal diagnóstico de ambos os grupos a síndrome do desconforto respiratório, durante as primeiras 48h pósextubação. Para análise estatística utilizou-se o test t Student, Mann- Whitney, Qui-quadrado e o teste exato de Fisher, com nível de significância em 5\%. A conclusão deste estudo foi que a manobra de AFE é menos estressante que a FRC em prematuros no período pós-extubação. Os resultados encontrados para tal afirmação denotaram que a FRC aumentou significativamente a FC, enquanto a manobra de AFE não demonstrou alteração.

Nicolau e Falcão (2010) analisaram as repercussões da fisioterapia respiratória e aspiração endotraqueal, sobre a função cardiopulmonar de 42 recém-nascidos 
pré-termo (RNPT), com peso de nascimento médio de $1024 \mathrm{~g}$ e idade gestacional média de 29,5 semanas, submetidos à ventilação mecânica. Sendo o principal diagnóstico respiratório (88\%) a Doença das Membranas Hialinas. Verificou-se os valores de frequência cardíaca (FC), saturação de oxigênio (SatO²), frequência respiratória $(F R)$ e pressão arterial sistêmica (PA) por meio da monitorização eletrônica e FR, por cronômetro. Para análise estatística, foi aplicado o teste de ANOVA para medidas repetidas, sendo significante $p<0,05$. Os achados destes autores demonstraram que os procedimentos de fisioterapia respiratória e de aspiração endotraqueal não apresentaram influências significativas na função cardiopulmonar, diante as repercussões positivas dos parâmetros avaliados, sugerindo que, quando bem indicados e realizados, não comprometem a estabilidade clínica de RNPT

Assumpção et al. (2013) verificaram as implicações da vibrocompressão manual e da aspiração nasotraqueal, em 20 lactentes, 10 em cada grupo, no pósoperatório de cirurgias cardíacas, sobre os parâmetros cardiorrespiratórios de FC e FR, SpO², dor (Neonatal Infant Pain Scale - NIPS) e desconforto respiratório (Boletim de Silvermann-Andersen - BSA). Sendo tratados os dados, por meio de análise de variância (ANOVA) para medidas repetidas, sendo significante $p \leq 0,05$. De acordo com estes autores, a vibrocompressão manual e a aspiração nasotraqueal aplicadas em lactentes no pós-operatório de cirurgias cardíacas não prejudicaram a SpO2 e a FR, além de não desencadearem dor e desconforto respiratório, haja vista a indiferença estatística em nenhum dos dados (SpO2 - $p=0,77$; $f c-p=0,14$; $f r-p=0,17$; NIPS $-p=0,49$ e BSA $-p=0,51$ ). Dando continuidade, Ribeiro, Melo e Davidson (2008), Moreno, Fernandes e Guerra (2011) e Vasconcelos, Almeida e Bezerra (2011) foram estudos teóricos. Ribeiro, Melo e Davidson (2008) buscaram revisar as complicações pulmonares e intervenções fisioterapêuticas disponíveis para recém-nascidos, com persistência do canal arterial (PCA), nas fontes de dados Medline e Lilacs, além de capítulos de livros nos idiomas português e inglês, entre 1992 e 2006. A síntese dos dados desses autores evidenciou que a PCA é uma intercorrência frequente em recém-nascidos pré-termos, que pode se relacionar com complicações pulmonares, por exemplo: atelectasias, infecções pulmonares e falha na extubação, bem como o aumento do tempo de ventilação mecânica e de internação na Unidade de Terapia Intensiva Neonatal. Ressaltaram que os recursos fisioterapêuticos objetivam melhorar as condições pulmonares, facilitando o aumento da complacência pulmonar e a diminuição da resistência de vias aéreas, além disso, visam aperfeiçoar o mecanismo de depuração mucociliar, consequentemente reduzindo o trabalho respiratório. Assim sendo, concluíram que a fisioterapia respiratória contribui para melhorar a evolução dos neonatos com complicações pulmonares por persistência de canal arterial, permitindo aperfeiçoar a mecânica respiratória, além de melhorar a oxigenação e a relação ventilação/perfusão.

Os autores Moreno, Fernandes e Guerra (2011) realizaram uma revisão de literatura, selecionando 29 artigos científicos, via PubMed e ISI Web, além de um capitulo de livro nacional, publicados entre 1986 e 2009, com o objetivo de ponderar o papel da fisioterapia motora, no prematuro com risco de desenvolver doença metabólica óssea. Sendo esta última, compreendida como um conjunto de condições relacionadas a alterações no processo de calcificação fisiológica, resultando desde a fragilidade estrutural até o desenvolvimento de fraturas. Tais autores concluíram que a implementação de exercícios de fisioterapia motora parece proporcionar estabilidade ou estímulo para a formação óssea, podendo, consequentemente, prevenir e/ou minimizar as complicações decorrentes da 
doença metabólica óssea, diante ao aumento na densidade e no conteúdo mineral ósseo, correlacionados à aplicação rotineira de exercícios de mobilização passiva articular, massagem e posicionamentos.

E por fim, Vasconcelos, Almeida e Bezerra (2011) avaliaram o impacto e os benefícios da intervenção do fisioterapeuta no desfecho dos recém-nascidos internados na UTIN do Hospital Agamenon Magalhães (HAM), sendo realizada uma análise de 195 prontuários de arquivos médicos e estatísticos (Same), por meio do programa SPSS versão 13.0 para Windows e Excel 2003. Como desfecho, os resultados obtidos demonstraram uma influência positiva da maior permanência do fisioterapeuta na UTIN.

Quadro 2 - Critérios de Intervenção Fisioterapêutica nas Unidades de Terapia Intensiva em Neonatologia.

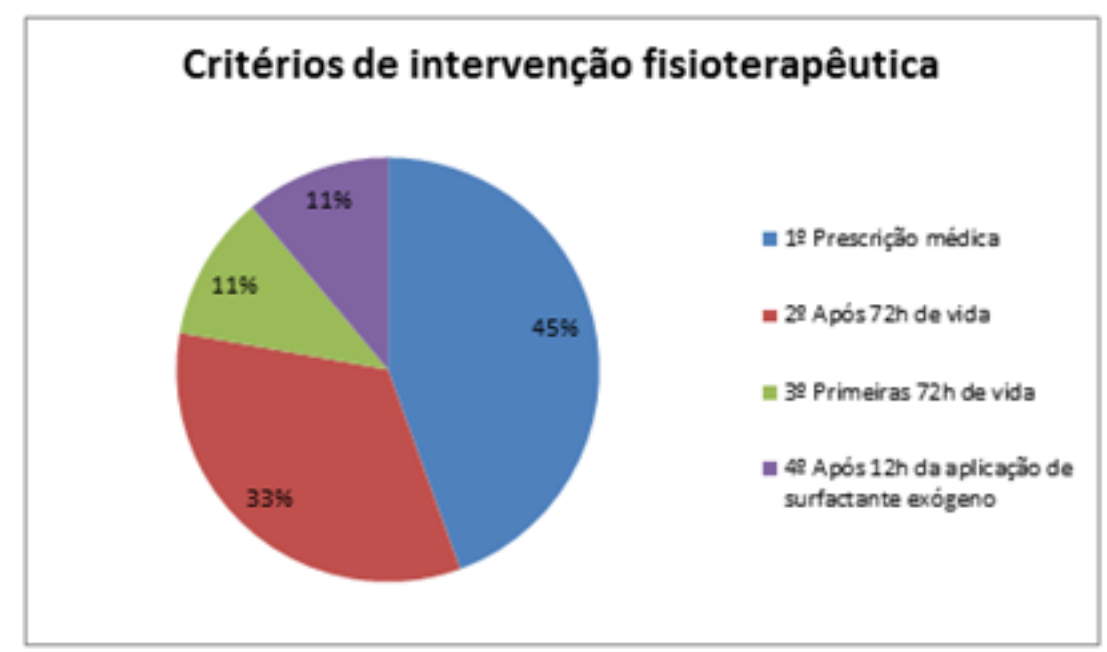

Fonte: Autores, 2017.

Referente aos critérios para início da intervenção fisioterapêutica, a Figura 2 demonstra os quatro principais fundamentos de indicação. Os autores Antunes et al. (2006), Vasconcelos, Almeida e Bezerra (2011), Assumpção et al. (2013) e Vignochi, Teixeira e Nader (2010) descrevem a indicação da fisioterapia somente após prescrição médica; Nicolau e Falcão (2010), Moreno, Fernandes e Guerra (2011) e Bassani et al. (2016) referem a indicação somente após 72 horas de vida; Ribeiro, Melo e Davidson (2008) indicam nas primeiras 72 horas de vida; e Cabral e Velloso (2014) mencionam o emprego do manuseio mínimo após 12 horas da aplicação do surfactante exógeno.

Condizente a prescrição médica, Antunes et al. (2006), Vignochi, Teixeira e Nader (2010) e Assumpção et al. (2013), justificam a indicação da intervenção fisioterapêutica a partir do momento em que os neonatos prematuros apresentarem estabilidade clínica. Na revisão de Vasconcelos, Almeida e Bezerra (2011) a indicação da fisioterapia varia de acordo com o perfil profissional médico e descrevem que existem locais em que todos os pacientes internados na UTIN recebem este tipo de atendimento.

Pertinente à indicação somente após 72 horas de vida, Nicolau e Falcão (2010) justificam este critério diante o viés de que a hemorragia intracraniana peri- 
intraventricular ocorre com maior frequência nas primeiras 72 horas de vida nos RNPT com peso de nascimento inferior a $1500 \mathrm{~g}$. Ressaltam ainda que inúmeros fatores intra e extravasculares podem contribuir para promover o sangramento ou o seu aumento, como exemplo citam: a persistência do canal arterial, a ventilação mecânica e, supostamente, alguns procedimentos como a aspiração traqueal e a própria fisioterapia respiratória.

Moreno, Fernandes e Guerra (2011) expõe que é imprescindível que o recémnascido tenha idade pós-natal maior que 72 horas, com a matriz germinativa periventricular, no cérebro, menos suscetível a sangramento, que esteja estável hemodinamicamente, apresentando ganho de peso e em ventilação espontânea (podendo fazer uso de oxigênio). Estes mesmo autores relatam que crianças com diagnóstico de displasia broncopulmonar e sepse neonatal tratada, que fizeram uso de alguns medicamentos (diuréticos, corticosteroides, xantinas ou sedativos) e nutrição parenteral, podem ser favorecidos pela intervenção da fisioterapia, por se tratar de grupo de risco para deficiência mineral óssea. Como fechamento, concluem que se faz necessário o consentimento da equipe médica e a discussão da importância assistencial para cada caso.

Ainda, corroborando com este critério temporal de indicação (após 72 horas de vida), Bassani et al. (2016) argumentam os possíveis efeitos diante a aplicação da manobra de AFE, já que esta envolve compressão, mesmo que leve e lenta, de um tórax mais complacente. Justificam que a pressão exercida sobre o abdômen possa aumentar significativamente a pressão intra-abdominal e, em consequência, a pressão intratorácica e a pressão intracraniana (PIC), porém, esta teoria ainda não foi confirmada.

Em contrapartida, Ribeiro, Melo e Davidson (2008) contemplam em seus argumentos técnicas que visam aumentar o fluxo expiratório, cujo principal objetivo visa gerar maior velocidade de fluxo de gás na fase expiratória, com o intuito de desprender as secreções aderidas na árvore brônquica. Descrevem que tais técnicas podem ser realizadas em neonatos submetidos à correção da persistência do canal arterial (PCA), hemodinamicamente estáveis. Todavia, se faz necessário verificar a presença de desconforto respiratório e/ou dor pósoperatório, buscando identificar o momento propício para a aplicabilidade. Salientam que, nos prematuros com idade gestacional <34 semanas e/ou $<1.500 \mathrm{~g}$, principalmente nas primeiras 72 horas de vida, as técnicas de higiene brônquica, como vibração torácica manual, drenagem postural, aumento do fluxo expiratório e aspiração, têm de serem realizadas com cautela, devido a maior suscetibilidade de hemorragia peri-intraventricular pela imaturidade vascular do sistema nervoso central. Além do mais, as manobras compressivas são evitadas, pois podem favorecer o colabamento das vias aéreas e diminuição do volume pulmonar, resultando em atelectasias.

Por fim, Cabral e Velloso (2014) mencionam o emprego do manuseio mínimo após 12 horas da aplicação do surfactante exógeno, sendo que a participação do fisioterapeuta envolveu os procedimentos de aspiração, reposicionamento do tubo endotraqueal, ajuste de ventilação mecânica e troca de fixação do tubo endotraqueal. Vale ressaltar que o manuseio mínimo foi realizado por uma equipe multiprofissional composta por médicos, enfermeiros e fisioterapeutas. Apesar da recomendação prática do manuseio mínimo, há carência de estudos na literatura que avaliem os efeitos da utilização desta prática nesses $\mathrm{RN}$, assim como, não se sabe o tempo necessário para garantir maior estabilidade fisiológica. Porém, os achados conclusivos deste estudo contribuem efetivamente para a atuação da equipe multiprofissional das UTIN, sobretudo para o fisioterapeuta, de maneira a executar procedimentos de avaliação e 
intervenção criteriosamente definida e baseada em evidência científica. Dessa forma, instigando a necessidade de esclarecer técnicas e procedimentos ainda não tão bem elucidados neste contexto.

\section{CONCLUSÃO}

A pesquisa demonstra que na literatura existem poucos critérios justificados para início da intervenção fisioterapêutica na UTI neonatal e revela que não existe um consenso entre instituições e profissionais para o início da intervenção. Entre os critérios encontrados na literatura, apenas um justifica a escolha do início da atuação com específica determinação em detrimento de condição fisiológica do neonato (após 72h), os demais que citam indicação médica não descrevem quais critérios esses profissionais utilizam, portanto, não respondem ao questionamento desta pesquisa. Mesmo com prescrição médica o fisioterapeuta deve estar inserido na equipe multiprofissional, participar das decisões terapêuticas e avaliar o momento para iniciar a intervenção em conjunto com esta equipe. Dessa forma, estes resultados ressaltam a necessidade de maior exploração e pesquisa quanto aos critérios de início da intervenção da fisioterapia com prematuros e o tipo de atuação (fisioterapia motora, respiratória ou ambas). Assim poderemos expandir a ciência da fisioterapia neonatal, ressaltar sua importância na área hospitalar e apoiar recomendações práticas a fim de elevar a qualidade e principalmente a segurança dos atendimentos aos lactentes prematuros.

\section{REFERÊNCIA}

ALMEIDA, Cm; ALMEIDA, Afn; FORTI, Emp. Efeitos do Método Mãe Canguru nos Sinais Vitais de Recém-Nascidos Pré-Termo de Baixo Peso. Revista Brasileira de Fisioterapia, [s.i.], v. 11, n. 1, p.1-5, fev. 2007.

ANTUNES, LCO et al. Efeitos da fisioterapia respiratória convencional versus aumento do fluxo expiratório na saturação de O2, frequência cardíaca e frequência respiratória, em prematuros no período pós-extubação. Revista Brasileira de Fisioterapia, [s.I.], v. 10, n. 1, p.97-103, 2006. FapUNIFESP (SciELO). http://dx.doi.org/10.1590/s1413-35552006000100013.

ASSUMPCAO, Maíra Seabra de et al . Vibrocompressão manual e aspiração nasotraqueal no pós-operatório de lactentes cardiopatas. Rev. Paul. Pediatr, São Paulo, v. 31, n. 4, p. 507-515, Dec. 2013. Available from <http://www.scielo.br/scielo.php?script=sci_arttext\&pid=S0103$05822013000400507 \&$ Ing=en\&nrm=iso $>$ acesso em 19 nov. 2017. http://dx.doi.org/10.1590/S0103-05822013000400014.

BALBI, Bruna; CARVALHAES, Maria Antonieta de Barros Leite; PARADA, Cristina Maria Garcia de Lima. Tendência temporal do nascimento pré-termo e de seus determinantes em uma década. Ciênc. Saúde Coletiva, [s.I.], v. 21, n. 1, p.233-241, jan. 2016. FapUNIFESP

(SciELO).http://dx.doi.org/10.1590/141381232015211.20512015. 
BASSANI, Mariana Almada et al. Cerebral blood flow assessment of preterm infants during respiratory therapy with the expiratory flow increase technique. Revista Paulista de Pediatria (english Edition), [s.I.], v. 34, n. 2, p.178-183, jun. 2016. Elsevier BV. http://dx.doi.org/10.1016/j.rppede.2016.02.007.

CABRAL, Laura A.; VELLOSO, Marcelo. Comparing the effects of minimal handling protocols on the physiological parameters of preterm infants receiving exogenous surfactant therapy. Braz. J. Phys. Ther., São Carlos, v. 18, n. 2, p. 152-164, Apr. 2014. Available from <http://www.scielo.br/scielo.php?script=sci_arttext\&pid=S141335552014000200152\&lng=en\&nrm=iso $>$. access on 19 Nov. 2017. http://dx.doi.org/10.1590/S1413-35552012005000154.

GUINSBURG, Ruth; ALMEIDA, Maria Fernanda Branco de (Org.).

Reanimação do Prematuro. 2016. Elaborado por Sociedade Brasileira de Pediatria. Disponível em:

<http://www.sbp.com.br/reanimacao/wpcontent/uploads/2016/01/DiretrizesSBP ReanimacaoPrematuroMenor34semanas26jan2016.pdf>. Acesso em: 18 set. 2016.

HACKBARTH, Bruna Barbosa et al. Revista Suscetibilidade à prematuridade: investigação de fatores comportamentais, genéticos, médicos e sociodemográficos. Brasileira de Ginecologia e Obstetrícia, [s.I.], v. 37, n. 8, p.353-358, ago. 2015. FapUNIFESP (SciELO). http://dx.doi.org/10.1590/so100 720320150005338 .

MARTINS, Renata et al. Técnicas de fisioterapia respiratória: efeito nos parâmetros cardiorrespiratórios e na dor do neonato estável em UTIN. Revista Brasileira de Saúde Materno Infantil, [s.I.], v. 13, n. 4, p.317-327, dez. 2013. FapUNIFESP (SciELO). http://dx.doi.org/10.1590/s1519-38292013000400004.

MORENO, Juliana; FERNANDES, Luciana Volpiano; GUERRA, Camila Campos. Fisioterapia motora no tratamento do prematuro com doença metabólica óssea. Rev. paul. pediatr., São Paulo, v. 29, n. 1, p. 117-121, Mar. 2011. Available from <http://www.scielo.br/scielo.php?script=sci_arttext\&pid=S010305822011000100018\&lng=en\&nrm=iso>. access on 19 Nov. 2017. http://dx.doi.org/10.1590/S0103-05822011000100018.

NICOLAU, Carla Marques; FALCAO, Mário Cícero. Influência da fisioterapia respiratória sobre a função cardiopulmonar em recém-nascidos de muito baixo peso. Rev. paul. pediatr., São Paulo, v. 28, n. 2, p. 170-175, June 2010. Available from <http://www.scielo.br/scielo.php?script=sci_arttext\&pid=S010305822010000200007\&lng=en\&nrm=iso >. access on 19 Nov. 2017. http://dx.doi.org/10.1590/S0103-05822010000200007.

RIBEIRO, Ivete Furtado; MELO, Ana Paula L. de; DAVIDSON, Josy. Fisioterapia em recém-nascidos com persistência do canal arterial e complicações pulmonares. Rev. paul. pediatr., São Paulo, v. 26, n. 1, p. 77-83, Mar. 2008. Available from 
<http://www.scielo.br/scielo.php?script=sci_arttext\&pid=S0103-

05822008000100013\&lng=en\&nrm=iso $>$. access on 19 Nov. 2017.

http://dx.doi.org/10.1590/S0103-05822008000100013.

SANTOS, Adriana Mello Rodrigues dos; MEIRA, Zilda Maria Alves; PEREIRA, Maria do Carmo Nunes. Echocardiography Role in Assessing Cardiovascular Changes in Very Low Birth Weight Babies, With Emphasis on the Presence of the Ductus Arteriosus. Arquivos Brasileiros de Cardiologia - Imagem Cardiovascular, [s.I.], v. 29, n. 2, p.47-57, jan. 2016. GN1 Genesis Network. http://dx.doi.org/10.5935/2318-8219.20160014.

SELESTRIN, Cláudia de Castro et al. Avaliação dos parâmetros fisiológicos em recém nascidos pré-termo em ventilação mecânica após procedimentos de fisioterapia neonatal. Rev Bras Crescimento Desenvolv Hum, São Paulo, v. 1, n. 17, p.146-155, nov. 2007.

VASCONCELOS, Gabriela Arruda Reinaux de; ALMEIDA, Rita de Cássia Albuquerque; BEZERRA, Andrezza de Lemos. Repercussões da fisioterapia na unidade de terapia intensiva neonatal. Fisioter. mov. (Impr.), Curitiba, v. 24 , n. 1, p. 65-73, Mar. 2011. Available from

$<$ http://www.scielo.br/scielo.php?script=sci_arttext\&pid=S0103-

$51502011000100008 \& \operatorname{lng}=e n \& n r m=i s o>$. access on 19 Nov. 2017. http://dx.doi.org/10.1590/S0103-51502011000100008.

VIGNOCHI, Carine; TEIXEIRA, Patrícia P.; NADER, Silvana S.. Efeitos da fisioterapia aquática na dor e no estado de sono e vigília de recémnascidos pré-termo estáveis internados em unidade de terapia intensiva neonatal. Revista Brasileira de Fisioterapia, [s.I.], v. 14, n. 3, p.214-220, jun. 2010. FapUNIFESP (SciELO). http://dx.doi.org/10.1590/s141335552010000300013. 